\title{
Logistic vector random fields with logistic direct and cross covariances
}

\author{
N. Balakrishnan ${ }^{\mathrm{a}}$, Chunsheng Ma ${ }^{\mathrm{b}, 1}$, Renxiang Wang ${ }^{\mathrm{c}}$ \\ ${ }^{a}$ Department of Mathematics and Statistics, McMaster University, Hamilton, Ontario, \\ Canada $L 8 K 4 K 1$ \\ ${ }^{b}$ Department of Mathematics, Statistics, and Physics, Wichita State University, Wichita, \\ KS 67260, USA \\ Hubei Engineering University, Xiaogan, Hubei 432000, China \\ ${ }^{c}$ School of Economics, Wuhan University of Technology, Wuhan, Hubei 430070, China
}

\section{Abstract}

The logistic vector random field is introduced in this paper as a scale mixture of Gaussian vector random fields, and is thus a particular elliptically contoured (spherically invariant) vector random field. Such a logistic vector random field is characterized by its mean function and covariance matrix function just as in the case of Gaussian vector random fields, and so it is flexible enough to allow for any possible mean structure or covariance matrix structure. We also derive covariance matrix functions whose direct and cross covariances are of the logistic type.

Keywords: Covariance matrix function, Elliptically contoured random field, Gaussian random field, Isotropic, Logistic distribution, Spherically invariant random field, Stationary, Variogram

\footnotetext{
${ }^{1}$ Corresponding author.

E-mail address: chunsheng.ma@wichita.edu (C. Ma)

Preprint submitted to Journal of Statistical Planning and Inference

October 13, 2014 


\section{Introduction}

The univariate logistic distribution and its multivariate forms have been studied quite extensively in the literature, and have also been used widely in practice ( Malik and Abraham (1973), Balakrishnan (1992), Arnold (1996), Kotz et al. (2000), Balakrishnan and Lai (2009)), just like the normal distribution. However, research on scalar (univariate) time series or stochastic processes with logistic marginal distributions has been rather minimal compared to the voluminous work that has been carried out on Gaussian time series or stochastic processes; see Mansfield and Hensley (1960), Arnold (1988), (1992), (1993), Arnold and Robertson (1989), Tan and Piantadosi (1991), Sim (1993), Grasman (1998), Newman et al. (2004), Soboleva and Pleasanta (2003), and Silva et al. (2005). Very few multiple time series or vector (multivariate) stochastic process with logistic marginals have been discussed in the literature. This motivates us to introduce vector logistic random fields in space and/or time, whose special features are that they allow for any possible mean structure or covariance matrix structure, just as the Gaussian random field does.

Suppose that $\left\{\mathbf{Z}(x)=\left(Z_{1}(x), \ldots, Z_{m}(x)\right)^{\prime}, x \in \mathbb{D}\right\}$ is an $m$-variate secondorder random field, or a set of $m$-variate real random vectors on the same probability space with finite second-order moments, where $\mathbb{D}$ is a temporal, spatial, or spatio-temporal index domain. Denote the covariance matrix function of $\{\mathbf{Z}(x), x \in \mathbb{D}\}$ by

$$
\mathbf{C}\left(x_{1}, x_{2}\right)=\mathrm{E}\left\{\left[\mathbf{Z}\left(x_{1}\right)-\mathrm{E} \mathbf{Z}\left(x_{1}\right)\right]\left[\mathbf{Z}\left(x_{2}\right)-\mathrm{E} \mathbf{Z}\left(x_{2}\right)\right]^{\prime}\right\}, \quad x_{1}, x_{2} \in \mathbb{D},
$$

where $\mathrm{E} \mathbf{Z}(x)$ is the expectation or mean of $\mathbf{Z}(x)$. A diagonal entry of the above covariance matrix function, $C_{i i}\left(x_{1}, x_{2}\right)$, is called a direct covariance (function), and an off-diagonal entry, $C_{i j}\left(x_{1}, x_{2}\right), i \neq j$, is called a cross covariance (function), $i, j=1, \ldots, m$. In particular, $\{\mathbf{Z}(x), x \in \mathbb{D}\}$ is said to be (weakly, or second-order) stationary if $\mathrm{EZ}(x)$ is identical to a vector and $\mathbf{C}\left(x_{1}, x_{2}\right)$ depends only on the lag $x_{1}-x_{2}$. In such a case, we write $\mathbf{C}\left(x_{1}, x_{2}\right)$ as $\mathbf{C}\left(x_{1}-x_{2}\right)$ for simplicity.

Two fundamental properties of $\mathbf{C}\left(x_{1}, x_{2}\right)$ are

(i) the transpose of $\mathbf{C}\left(x_{1}, x_{2}\right)$ equals $\mathbf{C}\left(x_{2}, x_{1}\right)$, i.e.,

$$
\left(\mathbf{C}\left(x_{1}, x_{2}\right)\right)^{\prime}=\mathbf{C}\left(x_{2}, x_{1}\right) ;
$$


(ii) the inequality

$$
\sum_{i=1}^{n} \sum_{j=1}^{n} \mathbf{a}_{i}^{\prime} \mathbf{C}\left(x_{i}, x_{j}\right) \mathbf{a}_{j} \geq 0
$$

holds for every natural number $n$, any $x_{i} \in \mathbb{D}$, and any $\mathbf{a}_{i} \in \mathbb{R}^{m}$, $i=1, \ldots, n$.

Conversely, for a given $m \times m$ matrix function $\mathbf{C}\left(x_{1}, x_{2}\right)$ on $\mathbb{D} \times \mathbb{D}$ (or on $\mathbb{D}$, for simplicity), if it satisfies the above two properties (i) and (ii), then there exists an $m$-variate Gaussian or elliptically contoured (spherically invariant) random field with mean $\mathbf{0}$ and with $\mathbf{C}\left(x_{1}, x_{2}\right)$ as its covariance matrix function; see, for instance, Cramér and Leadbetter (1967), Gikhman and Skorokhod (1969), Yaglom (1987), and Ma (2011a). However, there may not exist a non-Gaussian, such as a $\chi^{2}$ (Ma (2011b)), log-Gaussian, skew-Gaussian, or $\mathrm{K}$-distributed random field with $\mathbf{C}\left(x_{1}, x_{2}\right)$ as its covariance matrix function. The logistic vector random field developed here belongs to the family of elliptically contoured (spherically invariant) vector random fields (Du and Ma (2011), Ma (2011a)), and thus allows for any given correlation structure.

The rest of this paper is organized as follows. Section 2 provides a brief review for univariate and multivariate logistic distributions. There are many versions of multivariate logistic distributions available in the literature, but we adopt here only one form as finite-dimensional distributions of the logistic vector random field. Section 3 defines the logistic vector random field and discusses its basic properties. Some covariance matrix structures, particularly those with logistic direct and cross covariances, are derived in Section 4 for logistic vector random fields. Some concluding remarks are made in Section 5, while the proofs of Theorems 2-5 are finally presented in Section 6 .

\section{Univariate and multivariate logistic distributions}

This section presents a brief review of univariate and multivariate logistic distributions. For more details, we refer the reader to Balakrishnan (1992), Arnold (1996), Kotz et al. (2000), and Balakrishnan and Lai (2009).

A univariate logistic distribution function takes on the form

$$
\left\{1+\exp \left(-\frac{\pi}{\sqrt{3} \sigma}(z-\mu)\right)\right\}^{-1}, \quad z \in \mathbb{R},
$$


with density

$$
\frac{\pi}{\sqrt{3} \sigma} \frac{\exp (-\pi(z-\mu) /(\sqrt{3} \sigma))}{\{1+\exp (-\pi(z-\mu) /(\sqrt{3} \sigma))\}^{2}}, \quad z \in \mathbb{R}
$$

and has mean $\mu$ and standard deviation $\sigma$. In the particular case when $\mu=0$ and $\sigma=\frac{\pi}{\sqrt{3}}$, the associated random variable $Z$ is often called a standard logistic random variable, and has its distribution function as

$$
F(z)=\left(1+e^{-z}\right)^{-1}, \quad z \in \mathbb{R}
$$

density as

$$
f(z)=\frac{e^{-z}}{\left(1+e^{-z}\right)^{2}}, \quad z \in \mathbb{R}
$$

and characteristic function as

$$
\varphi(\omega)=\frac{\pi \omega}{\sinh (\pi \omega)}, \quad \omega \in \mathbb{R} .
$$

A useful property (Andrews and Mallows (1974), Ghosh et al. (2010)) of the standard logistic density is the following integral expression:

$$
f(z)=\frac{1}{\sqrt{2 \pi}} \int_{0}^{\infty} \exp \left(-\frac{z^{2}}{2 u^{2}}\right) f_{U}(u) \frac{d u}{u}, \quad z \in \mathbb{R},
$$

where

$$
f_{U}(u)= \begin{cases}2 u \sum_{n=1}^{\infty}(-1)^{n-1} n^{2} \exp \left(-\frac{n^{2} u^{2}}{2}\right), & u>0, \\ 0, & u \leq 0\end{cases}
$$

is the density of the Kolmogorov-Smirnov random variable $U$ with distribution function

$$
P(U<u)= \begin{cases}1+2 \sum_{n=1}^{\infty}(-1)^{n} \exp \left(-\frac{n^{2} u^{2}}{2}\right), & u>0 \\ 0, & u \leq 0\end{cases}
$$

In other words, a standard logistic random variable $Z$ has the same distribution as $U Y$, where $Y$ is a standard normal random variable, $U$ is a Kolmogorov-Smirnov random variable, and $Y$ and $U$ are independent. As a consequence of (2) and (3), we also obtain the following identity

$$
\int_{0}^{\infty} \exp \left(-\frac{1}{2} \omega u^{2}\right) f_{U}(u) d u=\frac{\pi \omega^{\frac{1}{2}}}{\sinh \left(\pi \omega^{\frac{1}{2}}\right)}, \quad \omega \geq 0,
$$


which becomes useful when dealing with the multivariate case later. This identity may be derived by using (3) to evaluate a standard logistic random variable's characteristic function and then comparing with (2).

There are many versions of multivariate logistic distributions; see Balakrishnan (1992), Arnold (1996), and Kotz et al. (2000), among others. Herein, we adopt the following definition. An $m$-variate random vector $\mathbf{Z}$ is said to be a logistic random vector, if it has the same distribution as $U \mathbf{Y}+\boldsymbol{\mu}$, where $\mathbf{Y}$ is an $m$-variate normal random vector with mean $\mathbf{0}$ and variancecovariance matrix $\Sigma, U$ is a Kolmogorov-Smirnov random variable, $\mathbf{Y}$ and $U$ are independent, and $\boldsymbol{\mu}$ is an $m$-valued (non-random) vector.

For a logistic random vector $\mathbf{Z}$, we have its mean vector as

$$
\mathrm{EZ}=\mathrm{E} U \mathrm{EY}+\boldsymbol{\mu}=\boldsymbol{\mu},
$$

and its variance-covariance matrix as

$$
\operatorname{cov}(\mathbf{Z}, \mathbf{Z})=\operatorname{cov}(U \mathbf{Y}+\boldsymbol{\mu}, U \mathbf{Y}+\boldsymbol{\mu})=\mathrm{E} U^{2} \boldsymbol{\Sigma}=\frac{\pi^{2}}{3} \boldsymbol{\Sigma}
$$

where $\mathrm{E} U^{2}$ is evaluated by using identity (5) as follows:

$$
\begin{aligned}
\mathrm{E} U^{2} & =\int_{0}^{\infty} u^{2} f_{U}(u) d u \\
& =-\left.2 \frac{\partial}{\partial \omega} \int_{0}^{\infty} \exp \left(-\frac{1}{2} \omega u^{2}\right) f_{U}(u) d u\right|_{\omega=0} \\
& =-\left.2 \frac{d}{d \omega} \frac{\pi \omega^{\frac{1}{2}}}{\sinh \left(\pi \omega^{\frac{1}{2}}\right)}\right|_{\omega=0} \\
& =\frac{\pi^{2}}{3} .
\end{aligned}
$$

Also, by the use of identity (5), we obtain the characteristic function of $\mathbf{Z}$ as follows:

$$
\begin{aligned}
\operatorname{Eexp}\left(\imath \boldsymbol{\omega}^{\prime} \mathbf{Z}\right) & =\exp \left(\imath \boldsymbol{\omega}^{\prime} \boldsymbol{\mu}\right) \operatorname{Eexp}\left(\imath \boldsymbol{\omega}^{\prime} U \mathbf{Y}\right) \\
& =\exp \left(\imath \boldsymbol{\omega}^{\prime} \boldsymbol{\mu}\right) \int_{0}^{\infty} \operatorname{E} \exp \left(\imath u \boldsymbol{\omega}^{\prime} \mathbf{Y}\right) f_{U}(u) d u \\
& =\exp \left(\imath \boldsymbol{\omega}^{\prime} \boldsymbol{\mu}\right) \int_{0}^{\infty} \exp \left(-\frac{1}{2} u^{2} \boldsymbol{\omega}^{\prime} \boldsymbol{\Sigma} \boldsymbol{\omega}\right) f_{U}(u) d u \\
& =\exp \left(\imath \boldsymbol{\omega}^{\prime} \boldsymbol{\mu}\right) \frac{\left(\boldsymbol{\omega}^{\prime} \boldsymbol{\Sigma} \boldsymbol{\omega}\right)^{\frac{1}{2}} \pi}{\sinh \left(\left(\boldsymbol{\omega}^{\prime} \boldsymbol{\Sigma} \boldsymbol{\omega}\right)^{\frac{1}{2}} \pi\right)}, \quad \boldsymbol{\omega} \in \mathbb{R}^{m}
\end{aligned}
$$


where $\imath$ is the imaginary unit. From the above expression, it is readily seen that a logistic random vector is completely determined by its mean vector and covariance matrix, just like a normal random vector. The distribution function of a logistic random vector $\mathbf{Z}$ is given by

$$
\begin{aligned}
& P\left(Z_{1}<z_{1}, \ldots, Z_{m}<z_{m}\right)=P\left(U Y_{1}+\mu_{1}<z_{1}, \ldots, U Y_{m}+\mu_{m}<z_{m}\right) \\
= & \int_{0}^{\infty} P\left(Y_{1}<\frac{z_{1}-\mu_{1}}{u}, \ldots, Y_{m}<\frac{z_{m}-\mu_{m}}{u}\right) f_{U}(u) d u
\end{aligned}
$$

which does not have a closed-form expression except in the case of $m=1$, with

$$
P\left(Z_{1}<z_{1}\right)=\left\{1+\exp \left(-\frac{z-\mu_{1}}{\sigma_{1}}\right)\right\}^{-1}, \quad z_{1} \in \mathbb{R}
$$

where $\sigma_{1}^{2}=\operatorname{var}\left(Y_{1}\right)$.

\section{Logistic vector random fields}

In this section we give the formal definition of a vector logistic random field and then discuss some of its properties. Suppose that $\{\mathbf{Y}(x), x \in \mathbb{D}\}$ is an $m$-variate Gaussian random field with mean $\mathbf{0}$ and covariance matrix function $\mathbf{C}\left(x_{1}, x_{2}\right), U$ is a Kolmogorov-Smirnov random variable with distribution (4) and is independent of $\{\mathbf{Y}(x), x \in \mathbb{D}\}$, and $\boldsymbol{\mu}(x)$ is an $m$-valued (non-random) function on $\mathbb{D}$. Then, we define an $m$-variate logistic random field by

$$
\mathbf{Z}(x)=\left(\mathrm{E} U^{2}\right)^{-\frac{1}{2}} U \mathbf{Y}(x)+\boldsymbol{\mu}(x), \quad x \in \mathbb{D} .
$$

The term logistic random field is quite apt since the random vector $\mathbf{Z}(x)$ follows an $m$-variate logistic distribution. For this second-order random field, we have its mean vector as $\boldsymbol{\mu}(x)$, and its direct and cross covariances as

$$
\begin{aligned}
\operatorname{cov}\left(Z_{i}\left(x_{1}\right), Z_{j}\left(x_{2}\right)\right) & =\left(\mathrm{E}^{2}\right)^{-1} \operatorname{cov}\left(U Y_{i}\left(x_{1}\right), U Y_{j}\left(x_{2}\right)\right) \\
& =\operatorname{cov}\left(Y_{i}\left(x_{1}\right), Y_{j}\left(x_{2}\right)\right) \\
& =C_{i j}\left(x_{1}, x_{2}\right), \quad x_{1}, x_{2} \in \mathbb{D}, i, j=1, \ldots, m ;
\end{aligned}
$$

that is, $\{\mathbf{Z}(x), x \in \mathbb{D}\}$ has the same covariance matrix function as $\{\mathbf{Y}(x), x \in$ $\mathbb{D}\}$. This is the reason why we put the coefficient, $\left(\mathrm{E} U^{2}\right)^{-\frac{1}{2}}$, in front of the right-hand side of (6).

In order to expose the finite-dimensional distributions of an $m$-variate logistic random field, we take a look at the characteristic function of the 
random vector $\left(\mathbf{Z}^{\prime}\left(x_{1}\right), \mathbf{Z}^{\prime}\left(x_{2}\right), \ldots, \mathbf{Z}^{\prime}\left(x_{n}\right)\right)^{\prime}$, for an arbitrary natural number $n$ and any $x_{k} \in \mathbb{D}(k=1,2, \ldots, n)$. It then follows from (5) and (6) that

$$
\begin{aligned}
& \operatorname{E} \exp \left(\imath \sum_{k=1}^{n} \mathbf{Z}^{\prime}\left(x_{k}\right) \boldsymbol{\omega}_{k}\right) \\
= & \exp \left(\imath \sum_{k=1}^{n} \boldsymbol{\mu}^{\prime}\left(x_{k}\right) \boldsymbol{\omega}_{k}\right) \operatorname{E} \exp \left\{\imath\left(\mathrm{E} U^{2}\right)^{-\frac{1}{2}} U \sum_{k=1}^{n} \mathbf{Y}^{\prime}\left(x_{k}\right) \boldsymbol{\omega}_{k}\right\} \\
= & \exp \left(\imath \sum_{k=1}^{n} \boldsymbol{\mu}^{\prime}\left(x_{k}\right) \boldsymbol{\omega}_{k}\right) \int_{0}^{\infty} \operatorname{E} \exp \left\{\imath\left(\mathrm{E} U^{2}\right)^{-\frac{1}{2}} u \sum_{k=1}^{n} \mathbf{Y}^{\prime}\left(x_{k}\right) \boldsymbol{\omega}_{k}\right\} f_{U}(u) d u \\
= & \exp \left(\imath \sum_{k=1}^{n} \boldsymbol{\mu}^{\prime}\left(x_{k}\right) \boldsymbol{\omega}_{k}\right) \int_{0}^{\infty} \exp \left(-\frac{u^{2}}{2 \mathrm{E} U^{2}} \sum_{i=1}^{n} \sum_{j=1}^{n} \boldsymbol{\omega}_{i}^{\prime} \mathbf{C}\left(x_{i}, x_{j}\right) \boldsymbol{\omega}_{j}\right) f_{U}(u) d u \\
= & \left.\left.\sqrt{3} \exp \left(\imath \sum_{k=1}^{n} \boldsymbol{\mu}^{\prime}\left(x_{k}\right) \boldsymbol{\omega}_{k}\right)\left(\sum_{i=1}^{n} \sum_{j=1}^{n} \boldsymbol{\omega}_{i}^{\prime} \mathbf{C}\left(x_{i}, x_{j}\right) \boldsymbol{\omega}_{j}\right)^{\frac{1}{2}}\right)^{\frac{1}{2}}\right\}^{-1}, \\
& \times\left\{\sinh \left(3 \sum_{i=1}^{n} \sum_{j=1}^{n} \boldsymbol{\omega}_{i}^{\prime} \mathbf{C}\left(x_{i}, x_{j}\right) \boldsymbol{\omega}_{j}\right)^{\prime}, \boldsymbol{\omega}_{n} \in \mathbb{R}^{m} .\right.
\end{aligned}
$$

From this expression, we see that an $m$-variate logistic random field is completely determined by its mean vector and covariance matrix functions, just like the Gaussian or another elliptically contoured vector random field with second-order moments. As a consequence, if $\left\{\mathbf{Z}_{1}(x), x \in \mathbb{D}\right\}$ and $\left\{\mathbf{Z}_{2}(x), x \in\right.$ $\mathbb{D}\}$ are two mean-zero logistic vector random fields with covariance matrix functions $\mathbf{C}_{1}\left(x_{1}, x_{2}\right) \neq \mathbf{C}_{2}\left(x_{1}, x_{2}\right)$, this implies that the distributions of $\left\{\mathbf{Z}_{1}(x), x \in \mathbb{D}\right\}$ and $\left\{\mathbf{Z}_{2}(x), x \in \mathbb{D}\right\}$ are necessarily different.

The logistic vector random field, being a particular elliptically contoured random field, has its covariance matrix function characterized by the following theorem, which is obtained as a consequence of Theorem 8 of Ma (2011a).

Theorem 1. For an m-variate logistic random field, its covariance matrix function $\mathbf{C}\left(x_{1}, x_{2}\right)$ possesses the following properties:

(i) $\left(\mathbf{C}\left(x_{1}, x_{2}\right)\right)^{\prime}=\mathbf{C}\left(x_{2}, x_{1}\right), x_{1}, x_{2} \in \mathbb{D}$; 
(ii) the inequality

$$
\sum_{i=1}^{n} \sum_{j=1}^{n} \mathbf{a}_{i}^{\prime} \mathbf{C}\left(x_{i}, x_{j}\right) \mathbf{a}_{j} \geq 0
$$

holds for every natural number $n$, any $x_{i} \in \mathbb{D}$ and any $\mathbf{a}_{i} \in \mathbb{R}^{m}$, $i=1,2, \ldots, n$.

Conversely, for a given $m \times m$ matrix function $\mathbf{C}\left(x_{1}, x_{2}\right)$ on $\mathbb{D}$ with the above properties (i) and (ii), there exists an m-variate logistic random field with mean $\mathbf{0}$ and with $\mathbf{C}\left(x_{1}, x_{2}\right)$ as its covariance matrix function.

\section{Logistic direct and cross covariance matrix functions}

For logistic vector random fields on an index domain $\mathbb{D}$, we construct some covariance matrix structures in this section, which involve a logistic density, distribution, or related function. Our construction method has two other ingredients or building blocks: a scalar variogram, and a conditionally negative definite matrix, which are reviewed below first.

For $m \geq 2$, an $m \times m$ symmetric matrix $\Theta=\left(\theta_{i j}\right)$ is said to be conditionally negative definite (Bapat and Raghaven (1997)), if the inequality

$$
\sum_{i=1}^{m} \sum_{j=1}^{m} a_{i} a_{j} \theta_{i j} \leq 0
$$

holds for any real numbers $a_{1}, \ldots, a_{m}$ subject to the condition $\sum_{k=1}^{m} a_{k}=0$. It is said to be negative definite if the above inequality holds with or without the restriction $\sum_{k=1}^{m} a_{k}=0$. Examples of conditionally negative definite matrices are

(i) a matrix with identical entries,

(ii) a matrix with entries $\theta_{i j}=\theta_{i}+\theta_{j}$,

(iii) a matrix with entries $\theta_{i j}=\max \left(\theta_{i}, \theta_{j}\right)$,

(iv) a matrix with entries $\theta_{i j}=\left|\theta_{i}-\theta_{j}\right|$, where $\theta_{1}, \ldots, \theta_{m}$ are real numbers. 
A scalar variogram $\gamma\left(x_{1}, x_{2}\right), x_{1}, x_{2} \in \mathbb{D}$, is an important measure for the dependence of a scalar random field with second-order increments (Cressie (1993), Chilés and Delfiner (1999)), and possesses the following properties:

(i) $\gamma\left(x_{1}, x_{2}\right) \geq 0, \gamma(x, x) \equiv 0$

(ii) for every $n \geq 2$, and any $x_{k} \in \mathbb{D}(k=1, \ldots, n)$, an $n \times n$ matrix with entries $\gamma\left(x_{i}, x_{j}\right)$ is conditionally negative definite.

In the next theorem, a covariance matrix function is formulated in terms of the following three ingredients or building blocks: a scalar variogram, a conditionally negative definite matrix, and a standard logistic density function $e^{-x}\left(1+e^{-x}\right)^{-2}, x \in \mathbb{R}$.

Theorem 2. Let $\alpha$ be a positive constant and $0<\alpha \leq \frac{1}{2}$. If $\gamma\left(x_{1}, x_{2}\right)$ is a scalar variogram on $\mathbb{D}$ and $\boldsymbol{\Theta}=\left(\theta_{i j}\right)$ is an $m \times m$ conditionally negative definite matrix with positive entries, then there exist two $m$-variate logistic random fields on $\mathbb{D}$, with

(i) the first one having direct and cross covariances

$$
C_{i j}\left(x_{1}, x_{2}\right)=\exp \left(-\left(\gamma\left(x_{1}, x_{2}\right)+\theta_{i j}\right)^{\alpha}\right)\left\{1+\exp \left(-\left(\gamma\left(x_{1}, x_{2}\right)+\theta_{i j}\right)^{\alpha}\right)\right\}^{-2},
$$

and

(iii) the second one having direct and cross covariances

$$
C_{i j}\left(x_{1}, x_{2}\right)=\left(\frac{e^{-\left(\gamma\left(x_{1}, x_{2}\right)+\theta_{i j}\right)^{\alpha}}}{1+e^{-\left(\gamma\left(x_{1}, x_{2}\right)+\theta_{i j}\right)^{\alpha}}}\right)^{n}, \quad x_{1}, x_{2} \in \mathbb{D}, i, j=1, \ldots, m,
$$

where $n$ is a natural number.

Denote the Euclidean norm and $\ell_{1}$-norm of $\mathbf{x} \in \mathbb{R}^{d}$ by $\|\mathbf{x}\|$ and $|\mathbf{x}|$, respectively. It is known that $\|\mathbf{x}\|$ and $|\mathbf{x}|$ are intrinsically stationary variograms (Cressie (1993), Chilés and Delfiner (1999)); that is, $\left\|\mathbf{x}_{1}-\mathbf{x}_{2}\right\|$ and $\left|\mathbf{x}_{1}-\mathbf{x}_{2}\right|, \mathbf{x}_{1}, \mathbf{x}_{2} \in \mathbb{R}^{d}$, are variograms. A covariance matrix function is constructed in Theorems 3 and 4 with the following three ingredients: the Euclidean norm $\|\mathbf{x}\|$ or $\ell_{1}$-norm $|\mathbf{x}|$, a conditionally negative definite matrix, 
and a standard logistic density function $e^{-x}\left(1+e^{-x}\right)^{-2}, x \in \mathbb{R}$.

Theorem 3. Let $\alpha$ be a constant with $0<\alpha \leq 1$. If $\Theta=\left(\theta_{i j}\right)$ is an $m \times m$ conditionally negative definite matrix with positive entries, then there exists an $m$-variate stationary logistic random field in $\mathbb{R}^{d}$, with direct and cross covariances

$$
\begin{aligned}
& C_{i j}(\mathbf{x})=\theta_{i j}^{-\frac{d}{2}} \exp \left(-\theta_{i j}^{-\frac{\alpha}{2}}\|\mathbf{x}\|^{\alpha}\right)\left\{1+\exp \left(-\theta_{i j}^{-\frac{\alpha}{2}}\|\mathbf{x}\|^{\alpha}\right)\right\}^{-2}, \\
& \mathbf{x} \in \mathbb{R}^{d}, i, j=1, \ldots, m .
\end{aligned}
$$

In the particular case $\alpha=1$, those direct/cross covariances in (10) reduce to

$$
\begin{array}{r}
C_{i j}(\mathbf{x})=\theta_{i j}^{-\frac{1}{2}} \exp \left(-\theta_{i j}^{-\frac{1}{2}}\|\mathbf{x}\|\right)\left\{1+\exp \left(-\theta_{i j}^{-\frac{1}{2}}\|\mathbf{x}\|\right)\right\}^{-2}, \\
\mathbf{x} \in \mathbb{R}^{d}, i, j=1, \ldots, m .
\end{array}
$$

Their univariate margins

$$
\begin{aligned}
& C_{i j}\left(0, \ldots, 0, x_{k}, 0, \ldots, 0\right)=\theta_{i j}^{-\frac{1}{2}} \exp \left(-\theta_{i j}^{-\frac{1}{2}} x_{k}\right)\left\{1+\exp \left(-\theta_{i j}^{-\frac{1}{2}} x_{k}\right)\right\}^{-2}, \\
& x_{k} \in \mathbb{R}, i, j=1, \ldots, m,
\end{aligned}
$$

are infinitely differentiable on $\mathbb{R}$, so that an associated $m$-variate stochastic process $\left\{\mathbf{Z}\left(0, \ldots, 0, x_{k}, 0, \ldots, 0\right), x_{k} \in \mathbb{R}\right\}$, or $\left\{\mathbf{Z}(\mathbf{x}), x_{k} \in \mathbb{R}\right\}$ with fixed $x_{j}(j \neq k, j=1, \ldots, m)$, is infinitely mean-square differentiable, $k=1, \ldots, m$. Since the directional derivatives of $C_{i j}(\mathbf{x})$ exist, an associated random field $\left\{\mathbf{Z}(\mathbf{x}), \mathbf{x} \in \mathbb{R}^{d}\right\}$ has mean square derivative in any direction. See Banerjee and Gelfand (2003) and Xue and Xiao (2011) for the definition and properties of mean square directional derivatives, and also for those of mean square total differentiability, which is in analogous to total differentiability of a function in $\mathbb{R}^{d}$ in ordinary multivariate calculus. The bivariate margins

$$
\begin{gathered}
C_{i j}\left(0, \ldots, 0, x_{k}, 0, \ldots, x_{l}, \ldots, 0\right) \\
=\theta_{i j}^{-\frac{1}{2}} \exp \left(-\theta_{i j}^{-\frac{1}{2}} \sqrt{x_{k}^{2}+x_{l}^{2}}\right)\left\{1+\exp \left(-\theta_{i j}^{-\frac{1}{2}} \sqrt{x_{k}^{2}+x_{l}^{2}}\right)\right\}^{-2}, \\
\left(x_{k}, x_{l}\right) \in \mathbb{R}^{2}, i, j=1, \ldots, m
\end{gathered}
$$


are not (totally) differentiable at the origin, since the partial derivatives exist and continuous for all $\left(x_{k}, x_{l}\right) \neq(0,0)$ :

$$
\begin{aligned}
& \frac{\partial}{\partial x_{k}} C_{i j}\left(0, \ldots, 0, x_{k}, 0, \ldots, x_{l}, \ldots, 0\right) \\
&= \frac{x_{k}}{\theta_{i j} \sqrt{x_{k}^{2}+x_{l}^{2}}} \frac{\exp \left(-\theta_{i j}^{-\frac{1}{2}} \sqrt{x_{k}^{2}+x_{l}^{2}}\right)\left\{1-\exp \left(-\theta_{i j}^{-\frac{1}{2}} \sqrt{x_{k}^{2}+x_{l}^{2}}\right)\right\}}{\left\{1+\exp \left(-\theta_{i j}^{-\frac{1}{2}} \sqrt{x_{k}^{2}+x_{l}^{2}}\right)\right\}^{3}} \\
&= \frac{\partial}{\partial x_{l}} C_{i j}\left(0, \ldots, 0, x_{k}, 0, \ldots, x_{l}, \ldots, 0\right) \\
& \theta_{i j} \sqrt{x_{k}^{2}+x_{l}^{2}} \frac{\exp \left(-\theta_{i j}^{-\frac{1}{2}} \sqrt{x_{k}^{2}+x_{l}^{2}}\right)\left\{1-\exp \left(-\theta_{i j}^{-\frac{1}{2}} \sqrt{x_{k}^{2}+x_{l}^{2}}\right)\right\}}{\left\{1+\exp \left(-\theta_{i j}^{-\frac{1}{2}} \sqrt{x_{k}^{2}+x_{l}^{2}}\right)\right\}^{3}} \\
&\left(x_{k}, x_{l}\right) \in \mathbb{R}^{2}, i, j=1, \ldots, m,
\end{aligned}
$$

but the partial derivatives do not exist at $\left(x_{k}, x_{l}\right)=(0,0)$, and an associated $m$-variate random field $\left\{\mathbf{Z}\left(0, \ldots, 0, x_{k}, 0, \ldots, x_{l}, \ldots 0\right),\left(x_{k}, x_{l}\right) \in \mathbb{R}^{2}\right\}$ is not mean-square (totally) differentiable on $\mathbb{R}^{2}$.

Theorem 4. Let $\alpha$ be a constant with $0<\alpha \leq \frac{1}{2}$. If $\boldsymbol{\Theta}=\left(\theta_{i j}\right)$ is an $m \times m$ conditionally negative definite matrix with positive entries, then there exists an $m$-variate stationary logistic random field in $\mathbb{R}^{d}$, with direct and cross covariances

$$
\begin{aligned}
C_{i j}(\mathbf{x})=\theta_{i j}^{-\frac{d}{2}} \exp \left(-\theta_{i j}^{\frac{\alpha}{2}}|\mathbf{x}|^{\alpha}\right)\{ & \left.1+\exp \left(-\theta_{i j}^{\frac{\alpha}{2}}|\mathbf{x}|^{\alpha}\right)\right\}^{-2}, \\
& \mathbf{x} \in \mathbb{R}^{d}, i, j=1, \ldots, m .
\end{aligned}
$$

A Type I generalized logistic distribution function is of the form $(1+$ $\left.e^{-x}\right)^{-\nu}, x \in \mathbb{R}$, where $\nu$ is a positive constant (see, e.g., Dubey (1969) and Balakrishnan (1992)). Based on the reciprocal of such a distribution function, along with a scalar variogram and a conditionally negative definite matrix, we build a covariance matrix structure in the following theorem.

Theorem 5. Let $\alpha$ be a positive constant with $0<\alpha \leq \frac{1}{2}$. If $\gamma\left(x_{1}, x_{2}\right)$ is a scalar variogram on $\mathbb{D}, \boldsymbol{\Theta}=\left(\theta_{i j}\right)$ is an $m \times m$ conditionally negative definite matrix with positive entries, and $\nu_{k}(x)(k=1, \ldots, m)$ are positive functions 
on $\mathbb{D}$, then there exists an $m$-variate logistic random field on $\mathbb{D}$ with direct and cross covariances

$$
\begin{gathered}
C_{i j}\left(x_{1}, x_{2}\right)=\Gamma\left(\nu_{i}\left(x_{1}\right)+\nu_{j}\left(x_{2}\right)\right)\left\{1+\exp \left(-\left(\gamma\left(x_{1}, x_{2}\right)+\theta_{i j}\right)^{\alpha}\right)\right\}^{\nu_{i}\left(x_{1}\right)+\nu_{j}\left(x_{2}\right)} \\
x_{1}, x_{2} \in \mathbb{D}, i, j=1, \ldots, m .
\end{gathered}
$$

Here, $\Gamma(x)$ is Euler's Gamma function. One may be curious about the coefficient $\Gamma\left(\nu_{i}\left(x_{1}+\nu_{j}\right)\right.$ in (12) and wonder if it could be dropped and still have the remaining matrix function as a covariance matrix function. The following corollary is obtained by assuming that $\nu_{k}(x)(k=1, \ldots, m)$ are identical to positive constants.

Corollary. Given positive constants $\nu_{k}(k=1, \ldots, m)$, there exists an $m$ variate logistic random field on $\mathbb{D}$ with direct and cross covariances

$$
\begin{array}{r}
C_{i j}\left(x_{1}, x_{2}\right)=\Gamma\left(\nu_{i}+\nu_{j}\right)\left\{1+\exp \left(-\left(\gamma\left(x_{1}, x_{2}\right)+\theta_{i j}\right)^{\alpha}\right)\right\}^{\nu_{i}+\nu_{j}}, \\
x_{1}, x_{2} \in \mathbb{D}, i, j=1, \ldots, m .
\end{array}
$$

\section{Concluding remarks}

There are several versions of multivariate logistic distributions, such as those reviewed in Chapter 51 of Kotz et al. (2000), with which as finitedimensional distributions, one may define scalar logistic random fields. For example, by Kolmogorov's existence theorem, one obtains a scalar random field $\{Z(x), x \in \mathbb{D}\}$ with finite-dimensional logistic distributions of GumbelMalik-Abraham type (Malik and Abraham (1973)) of the form

$P\left(Z\left(x_{1}\right) \leq z_{1}, \ldots, Z\left(x_{n}\right) \leq z_{n}\right)=\left\{1+\sum_{k=1}^{n} \exp \left(-\frac{z_{k}-\mu\left(x_{k}\right)}{\sigma\left(x_{k}\right)}\right)\right\}^{-1}, \quad \mathbf{z} \in \mathbb{R}^{n}$,

where $\mu(x)$ and $\sigma(x), x \in \mathbb{D}$, are real functions with $\sigma(x)>0, n$ is an arbitrary natural number, and $x_{k} \in \mathbb{D}(k=1, \ldots, n)$. Alternatively, this random field may be formulated as

$$
Z(x)=\left\{Y(x)-Y_{0}\right\} \sigma(x)+\mu(x), \quad x \in \mathbb{D},
$$

where $\{Y(x), x \in \mathbb{D}\}$ is a set of independent and identically distributed extreme value random variables with density $\exp \{-y-\exp (-y)\}, y \in \mathbb{R}$, $Y_{0}$ an extreme value random variable with the same density, and $Y_{0}$ and 
$\{Y(x), x \in \mathbb{D}\}$ are independent. An obvious drawback of this random field is that its covariance function is always positive, but without flexible correlation. Specifically, we have

$$
\operatorname{cov}\left(Z\left(x_{1}\right), Z\left(x_{2}\right)\right)=\sigma\left(x_{1}\right) \sigma\left(x_{2}\right) \operatorname{var}\left(Y_{0}\right)=\frac{\pi^{2}}{6} \sigma\left(x_{1}\right) \sigma\left(x_{2}\right), \quad x_{1}, x_{2} \in \mathbb{D} .
$$

With the same drawback, another example is a scalar random field $\{Z(x), x \in$ $\mathbb{D}\}$ with finite-dimensional distributions of the form

$P\left(Z\left(x_{1}\right) \leq z_{1}, \ldots, Z\left(x_{n}\right) \leq z_{n}\right)=\left\{1+\left(\sum_{k=1}^{n} \exp \left(-\frac{z_{k}-\mu\left(x_{k}\right)}{\sigma\left(x_{k}\right)}\right)\right)^{\frac{1}{\alpha}}\right\}^{-1}, \quad \mathbf{z} \in \mathbb{R}^{n}$,

where $\alpha(\geq 1)$ is a constant. The last example is a scalar random field $\{Z(x), x \in \mathbb{D}\}$ with finite-dimensional distributions of the form

$P\left(Z\left(x_{1}\right) \leq z_{1}, \ldots, Z\left(x_{n}\right) \leq z_{n}\right)=\exp \left[-\left\{\sum_{k=1}^{n}\left(\ln \left(1+e^{-\frac{z_{k}-\mu\left(x_{k}\right)}{\sigma\left(x_{k}\right)}}\right)\right)^{\frac{1}{\alpha}}\right\}^{\alpha}\right], \quad \mathbf{z} \in \mathbb{R}^{n}$,

where $\alpha$ is a constant with $0<\alpha \leq 1$. It will naturally be of interest to see how the above multivariate logistic distributions could be used to introduce a logistic vector random field.

We have adopted here the approach of Ma (2011a) to construct logistic vector random fields, as compared to the above approach for the scalar case or those found in Mansfield and Hensley (1960), Arnold (1988), (1992), (1993), Arnold and Robertson (1989), Tan and Piantadosi (1991), Gerr and Allen (1993), Sim (1993), Grasman (1998), Soboleva and Pleasanta (2003), Newman at al. (2004), Silva et al. (2005), and Comellia et al. (2008). As in the Gaussian one, the logistic vector random field developed here allows for any possible mean structure or covariance matrix structure. This flexibility would prove quite useful while fitting models for practical data. Of course, the covariance matrix functions constructed here may be associated with not only a logistic vector random field but also Gaussian or other secondorder elliptically contoured vector random fields. Some brief discussions and comments are provided below for properties of our proposed covariance matrix structures, such as long-range dependence, stationarity, separability, and smoothness.

Short range dependence in vector random fields may be captured (modeled) by those covariance matrix functions in Theorems 3 and 4, since they are 
asymptotically equal to $\theta_{i j}^{-\frac{d}{2}} \exp \left(-\theta_{i j}^{-\frac{\alpha}{2}}\|\mathbf{x}\|^{\alpha}\right)$ or $\theta_{i j}^{-\frac{d}{2}} \exp \left(-\theta_{i j}^{\frac{\alpha}{2}}|\mathbf{x}|^{\alpha}\right)$. Short or long range dependence may be captured (modeled) by those covariance matrix functions in Theorem 2 and Corollary of Theorem 5, based on whether $\gamma\left(x_{1}, x_{2}\right)$ are unbounded or bounded. For example, in (8) taking $\gamma\left(x_{1}, x_{2}\right)=$ $1-\cos \left(x_{1}-x_{2}\right), x_{1}, x_{2} \in \mathbb{R}$, yields a long range dependent covariance matrix function.

Stationarity or nonstationarity of vector random fields may be captured (modeled) by those covariance matrix functions in Theorems 2 and 5, based on whether $\nu_{k}(x)(k=1, \ldots, m)$ are identical to constants and/or whether the scalar variogram $\gamma\left(x_{1}, x_{2}\right)$ is either intrinsically stationary or not.

When $\gamma\left(x_{1}, x_{2}\right)$ is a spatio-temporal variogram, some nonseparable spatiotemporal covariance matrix structures are provided in Theorems 2 and 5 for vector random fields on a spatio-temporal domain $\mathbb{D}$. See also Ma (2011b) for other nonseparable spatio-temporal covariance matrix structures.

If $\gamma\left(\mathbf{x}_{1}, \mathbf{x}_{2}\right)$ is a quadratic form, such as $\left\|\mathbf{A}\left(\mathbf{x}_{1}-\mathbf{x}_{2}\right)\right\|^{2}$, where $\mathbf{A}$ is a matrix with $d$ columns, then covariance matrix functions in Theorem 2 and in Corollary of Theorem 5 are infinitely differentiable, so that the associated vector random field is infinitely mean-square differentiable. So is the vector random field associated with the covariance matrix function (14) in the proof of Theorem 3. However, this may not be the case if $\gamma\left(\mathbf{x}_{1}, \mathbf{x}_{2}\right)$ is not a function of a quadratic form. When dealing with mean square differentiability of vector random fields, it is important to distinguish between the directional differentiability and the total differentiability, just like those in ordinary multivariate calculus.

\section{Proofs of main results}

\subsection{Proof of Theorem 2}

(i) Let $\beta=2 \alpha$. Clearly, $0<\beta \leq 1$. Using identity (3), we can rewrite (8) as

$$
\begin{aligned}
C_{i j}\left(x_{1}, x_{2}\right) & =\exp \left(-\left(\gamma\left(x_{1}, x_{2}\right)+\theta_{i j}\right)^{\frac{\beta}{2}}\right)\left\{1+\exp \left(-\left(\gamma\left(x_{1}, x_{2}\right)+\theta_{i j}\right)^{\frac{\beta}{2}}\right)\right\}^{-2} \\
& =\frac{1}{\sqrt{2 \pi}} \int_{0}^{\infty} \exp \left(-\frac{1}{2 u^{2}}\left(\gamma\left(x_{1}, x_{2}\right)+\theta_{i j}\right)^{\beta}\right) f_{U}(u) \frac{d u}{u} \\
& =\frac{\beta}{2 \sqrt{2 \pi}} \int_{0}^{\infty} \exp \left(-\left(\frac{\gamma\left(x_{1}, x_{2}\right)+\theta_{i j}}{v}\right)^{\beta}\right) f_{U}\left(\left(\frac{v^{\beta}}{2}\right)^{\frac{1}{2}}\right) \frac{d v}{v}
\end{aligned}
$$




$$
=\frac{\beta}{2 \sqrt{2 \pi}} \int_{0}^{\infty} C_{i j}\left(x_{1}, x_{2} ; v\right) f_{U}\left(\left(\frac{v^{\beta}}{2}\right)^{\frac{1}{2}}\right) \frac{d v}{v}
$$

where the third equation is obtained by making a substitution $v=\left(2 u^{2}\right)^{\frac{1}{\beta}}$, and

$$
C_{i j}\left(x_{1}, x_{2} ; v\right)=\exp \left\{-\left(\frac{\gamma\left(x_{1}, x_{2}\right)+\theta_{i j}}{v}\right)^{\beta}\right\}, \quad x_{1}, x_{2} \in \mathbb{D}, i, j=1, \ldots, m .
$$

By Theorem 4 of Ma (2011b), it now suffices to show that, for each fixed $v>0, C_{i j}\left(x_{1}, x_{2} ; v\right), i, j=1, \ldots, m$, form a covariance matrix function on $\mathbb{D}$.

While $0<\beta \leq 1$, it is shown in Pollard (1946) that the function $\exp \left(-x^{\beta}\right), x \geq 0$, is the Laplace transform of a distribution function, $G_{\beta}(\omega)$, say, with support on the interval $[0, \infty)$; that is,

$$
\exp \left(-x^{\beta}\right)=\int_{0}^{\infty} \exp (-x \omega) d G_{\beta}(\omega), \quad x \geq 0
$$

Using this identity, we rewrite $C_{i j}\left(x_{1}, x_{2} ; v\right)$ as

$$
\begin{array}{r}
C_{i j}\left(x_{1}, x_{2} ; v\right)=\int_{0}^{\infty} \exp \left(-\frac{\omega}{v} \gamma\left(x_{1}, x_{2}\right)\right) \exp \left(-\frac{\omega}{v} \theta_{i j}\right) d G_{\beta}(\omega), \\
x_{1}, x_{2} \in \mathbb{D}, i, j=1, \ldots, m .
\end{array}
$$

Since $\gamma\left(x_{1}, x_{2}\right)$ is a scalar variogram, $\exp \left(-\frac{\omega}{v} \gamma\left(x_{1}, x_{2}\right)\right), x_{1}, x_{2} \in \mathbb{D}$, is a covariance function for every fixed $\omega \geq 0$ and $v>0$, by Schoenberg's theorem (see, e.g., Ma (2005)). An $m \times m$ matrix with entries $\exp \left(-\frac{\omega}{v} \theta_{i j}\right)$ is positive definite, since $\Theta$ is conditionally negative definite. Thus, an $m \times m$ matrix function with entries $\exp \left(-\frac{\omega}{v} \gamma\left(x_{1}, x_{2}\right)\right) \exp \left(-\frac{\omega}{v} \theta_{i j}\right), x_{1}, x_{2} \in \mathbb{D}$, is a covariance matrix function. Moreover, by Theorem 4 of Ma (2011b), the $m \times m$ matrix function with entries $C_{i j}\left(x_{1}, x_{2} ; v\right)$ is a covariance matrix function.

(ii) In a way similar to that in the proof of Part (i), it can be shown that an $m \times m$ matrix function with entries $\exp \left(-\left(\gamma\left(x_{1}, x_{2}\right)+\theta_{i j}\right)^{\alpha}\right)$ is a covariance matrix function. An $m \times m$ matrix with all entries as 1 is obviously a covariance matrix function, and so its summation with the matrix function with entries $\exp \left(-\left(\gamma\left(x_{1}, x_{2}\right)+\theta_{i j}\right)^{\alpha}\right)$ is a covariance matrix function by Theorem 1 of Ma (2011b). This results in a covariance matrix function with entries $1+\exp \left(-\left(\gamma\left(x_{1}, x_{2}\right)+\theta_{i j}\right)^{\alpha}\right)$. Its Hadamard product with (8) is also a covariance matrix function by Theorem 3 of Ma (2011b), and so is the matrix function with entries (9). 


\subsection{Proof of Theorem 3}

We start by showing that the $m \times m$ matrix function

$$
\left(\begin{array}{cccc}
\theta_{11}^{-\frac{d}{2}} \exp \left(-\frac{\|\mathbf{x}\|^{2}}{\theta_{11}} u\right) & \theta_{12}^{-\frac{d}{2}} \exp \left(-\frac{\|\mathbf{x}\|^{2}}{\theta_{12}} u\right) & \cdots & \theta_{1 m}^{-\frac{d}{2}} \exp \left(-\frac{\|\mathbf{x}\|^{2}}{\theta_{1 m}} u\right) \\
\theta_{12}^{-\frac{d}{2}} \exp \left(-\frac{\|\mathbf{x}\|^{2}}{\theta_{12}} u\right) & \theta_{22}^{-\frac{d}{2}} \exp \left(-\frac{\|\mathbf{x}\|^{2}}{\theta_{22}} u\right) & \cdots & \theta_{2 m}^{-\frac{d}{2}} \exp \left(-\frac{\|\mathbf{x}\|^{2}}{\theta_{2 m}} u\right) \\
\vdots & \vdots & \ddots & \vdots \\
\theta_{1 m}^{-\frac{d}{2}} \exp \left(-\frac{\|\mathbf{x}\|^{2}}{\theta_{1 m}} u\right) & \theta_{2 m}^{-\frac{d}{2}} \exp \left(-\frac{\|\mathbf{x}\|^{2}}{\theta_{2 m}} u\right) & \cdots & \theta_{m m}^{-\frac{d}{2}} \exp \left(-\frac{\|\mathbf{x}\|^{2}}{\theta_{m m}} u\right)
\end{array}\right), \mathbf{x} \in \mathbb{R}^{d}
$$

is a covariance matrix function for each fixed $u>0$. To this end, we look at its corresponding Fourier transform matrix, which is positively proportional to

$$
\left(\begin{array}{cccc}
\exp \left(-\theta_{11}\|\boldsymbol{\omega}\|^{2} u^{-1}\right) & \exp \left(-\theta_{12}\|\boldsymbol{\omega}\|^{2} u^{-1}\right) & \cdots & \exp \left(-\theta_{1 m}\|\boldsymbol{\omega}\|^{2} u^{-1}\right) \\
\exp \left(-\theta_{12}\|\boldsymbol{\omega}\|^{2} u^{-1}\right) & \exp \left(-\theta_{22}\|\boldsymbol{\omega}\|^{2} u^{-1}\right) & \cdots & \exp \left(-\theta_{2 m}\|\boldsymbol{\omega}\|^{2} u^{-1}\right) \\
\cdots & \cdots & \cdots & \cdots \\
\exp \left(-\theta_{m 1}\|\boldsymbol{\omega}\|^{2} u^{-1}\right) & \exp \left(-\theta_{m 2}\|\boldsymbol{\omega}\|^{2} u^{-1}\right) & \cdots & \exp \left(-\theta_{m m}\|\boldsymbol{\omega}\|^{2} u^{-1}\right)
\end{array}\right), \boldsymbol{\omega} \in \mathbb{R}^{d}
$$

This matrix is positive definite for each fixed $\omega \in \mathbb{R}^{d}$, due to the assumption that $\Theta$ is conditionally negative definite. According to the CramérKolmogorov theorem (Cramér (1940)), (14) is a covariance matrix function.

Next, we shall show that an $m \times m$ matrix with entries

$$
\theta_{i j}^{-\frac{d}{2}} \exp \left(-\frac{\|\mathbf{x}\|^{2 \alpha}}{\theta_{i j}^{\alpha}} u^{\alpha}\right), \quad \mathbf{x} \in \mathbb{R}^{d}, i, j=1, \ldots, m,
$$

is a covariance matrix function for each fixed $u>0$. For this purpose, we use identity (13) to rewrite (15) as follows:

$\theta_{i j}^{-\frac{d}{2}} \exp \left(-\frac{\|\mathbf{x}\|^{2 \alpha}}{\theta_{i j}^{\alpha}} u^{\alpha}\right)=\theta_{i j}^{-\frac{d}{2}} \int_{0}^{\infty} \exp \left(-\frac{\|\mathbf{x}\|^{2}}{\theta_{i j}} u \omega\right) d G_{\alpha}(\omega), \quad \mathbf{x} \in \mathbb{R}^{d}, i, j=1, \ldots, m$.

Then, by Theorem 4 of Ma (2011b), (15) is a covariance matrix function since (14) is so.

Finally, we use identity (3) to rewrite $C_{i j}(\mathbf{x})$ of (10) as

$$
C_{i j}(\mathbf{x})=\frac{1}{\sqrt{2 \pi}} \theta_{i j}^{-\frac{d}{2}} \int_{0}^{\infty} \exp \left(-\frac{\|\mathbf{x}\|^{2 \alpha}}{2 \theta_{i j}^{\alpha} v^{2}}\right) f_{U}(v) \frac{d v}{v}, \quad \mathbf{x} \in \mathbb{R}^{d}, i, j=1, \ldots, m .
$$

By Theorem 4 of Ma (2011b) again, it is a covariance matrix function since (15) is a covariance matrix function for $u=\left(2 v^{2}\right)^{-\frac{1}{\alpha}}$. 


\subsection{Proof of Theorem 4}

For each fixed $u \geq 0$, the functions

$$
\theta_{i j}^{-\frac{d}{2}} \exp \left(-\theta_{i j}^{\alpha}|\mathbf{x}|^{2 \alpha} u\right), \quad \mathbf{x} \in \mathbb{R}^{d}, i, j=1, \ldots, m,
$$

form a covariance matrix function, as is shown in the proof of Theorem 4, Ma (2013). Using identities (3) and (13), we rewrite (11) as

$$
\begin{aligned}
C_{i j}(\mathbf{x})=\frac{1}{\sqrt{2 \pi}} \theta_{i j}^{-\frac{d}{2}} \int_{0}^{\infty} \exp \left(-\frac{\theta_{i j}^{\alpha}|\mathbf{x}|^{2 \alpha}}{2 u^{2}}\right) f_{U}(u) \frac{d u}{u}, & \\
& \quad \mathbf{x} \in \mathbb{R}^{d}, i, j=1, \ldots, m,
\end{aligned}
$$

which form a covariance matrix function, according to Theorem 4 of Ma (2011b).

\subsection{Proof of Theorem 5}

For a positive number $\nu$, it is known that the function $(1-x)^{-\nu},-1<$ $x<1$, has the following series expansion:

$$
(1-x)^{-\nu}=\sum_{n=0}^{\infty} \frac{\Gamma(\nu+n)}{\Gamma(\nu)} x^{n}, \quad-1<x<1
$$

see, for instance, (10.4.6) of Hansen (1975). Using (16), we rewrite (12) as

$$
\begin{gathered}
C_{i j}\left(x_{1}, x_{2}\right)=\Gamma\left(\nu_{i}\left(x_{1}\right)+\nu_{j}\left(x_{2}\right)\right)\left\{1-\frac{e^{-\left(\gamma\left(x_{1}, x_{2}\right)+\theta_{i j}\right)^{\alpha}}}{1+e^{-\left(\gamma\left(x_{1}, x_{2}\right)+\theta_{i j}\right)^{\alpha}}}\right\}^{-\nu_{i}-\nu_{j}} \\
=\sum_{n=0}^{\infty} \Gamma\left(\nu_{i}\left(x_{1}\right)+\nu_{j}\left(x_{2}\right)+n\right)\left\{\frac{e^{-\left(\gamma\left(x_{1}, x_{2}\right)+\theta_{i j}\right)^{\alpha}}}{1+e^{-\left(\gamma\left(x_{1}, x_{2}\right)+\theta_{i j}\right)^{\alpha}}}\right\}^{n}, \\
x_{1}, x_{2} \in \mathbb{D}, i, j=1, \ldots, m .
\end{gathered}
$$

In order to apply Theorems 1-3 of Ma (2011b) to show that the matrix with entries $C_{i j}\left(x_{1}, x_{2}\right)$ is a covariance matrix function, we just need to prove that, for each nonnegative integer $n$, an $m \times m$ matrix function with entries $\Gamma\left(\nu_{i}\left(x_{1}\right)+\nu_{j}\left(x_{2}\right)+n\right)$ is a covariance matrix function, since the matrix with entries

$$
\left\{\frac{e^{-\left(\gamma\left(x_{1}, x_{2}\right)+\theta_{i j}\right)^{\alpha}}}{1+e^{-\left(\gamma\left(x_{1}, x_{2}\right)+\theta_{i j}\right)^{\alpha}}}\right\}^{n}, \quad x_{1}, x_{2} \in \mathbb{D}, i, j=1, \ldots, m
$$


is a covariance matrix function by Part (ii) of Theorem 2. In fact, for every natural number $N$, and for any $\mathbf{a}_{k}=\left(a_{k 1}, \ldots, a_{k m}\right)^{\prime} \in \mathbb{R}^{m}$ and $x_{k} \in \mathbb{D}$ $(k=1, \ldots, N)$, we have

$$
\begin{aligned}
& \sum_{i=1}^{N} \sum_{j=1}^{N} \mathbf{a}_{i}^{\prime}\left(\begin{array}{ccc}
\Gamma\left(\nu_{1}\left(x_{i}\right)+\nu_{1}\left(x_{j}\right)+n\right) & \cdots & \Gamma\left(\nu_{1}\left(x_{i}\right)+\nu_{m}\left(x_{j}\right)+n\right) \\
\Gamma\left(\nu_{2}\left(x_{i}\right)+\nu_{1}\left(x_{j}\right)+n\right) & \cdots & \Gamma\left(\nu_{2}\left(x_{i}\right)+\nu_{m}\left(x_{j}\right)+n\right) \\
\ldots & \cdots & \cdots \\
\Gamma\left(\nu_{m}\left(x_{i}\right)+\nu_{1}\left(x_{j}\right)+n\right) & \cdots & \Gamma\left(\nu_{m}\left(x_{i}\right)+\nu_{m}\left(x_{j}\right)+n\right)
\end{array}\right) \mathbf{a}_{j} \\
= & \sum_{i=1}^{N} \sum_{j=1}^{N} \sum_{k=1}^{m} \sum_{l=1}^{m} a_{i k} \Gamma\left(\nu_{k}\left(x_{i}\right)+\nu_{l}\left(x_{j}\right)+n\right) a_{j l} \\
= & \sum_{i=1}^{N} \sum_{j=1}^{N} \sum_{k=1}^{m} \sum_{l=1}^{m} a_{i k} a_{j l} \int_{0}^{\infty} u^{\nu_{k}\left(x_{i}\right)+\nu_{l}\left(x_{j}\right)-1} e^{-u} d u \\
= & \int_{0}^{\infty}\left(\sum_{i=1}^{N} \sum_{k=1}^{m} a_{i k} u^{\nu_{k}\left(x_{i}\right)-\frac{1}{2}}\right)^{2} e^{-u} d u \\
\geq & 0,
\end{aligned}
$$

which implies that an $m \times m$ matrix function with entries $\Gamma\left(\nu_{i}\left(x_{1}\right)+\nu_{j}\left(x_{2}\right)+n\right)$ is a covariance matrix function by Theorem 8 of Ma (2011a).

Acknowledgments The authors wish to thank an associate editor and two anonymous reviewers for their valuable comments and suggestions which helped to improve the presentation of this paper. Ma's work was supported in part by U.S. Department of Energy under Grant DE-SC0005359.

\section{References}

Andrews, D. F., Mallows, C. F. 1974. Scale mixtures of normal distributions. Journal of the Royal Statistical Society, Series B 36, 99-102.

Arnold, B. C. 1988. A logistic process constructed using geometric minimization, Statistics and Probability Letters 7, 253-257.

Arnold, B. C. 1992. Logistic and semi-logistic processes, Journal of Computational and Applied Mathematics 40, 139-149. 
Arnold, B. C. 1993. Logistic processes involving Markovian minimization. Communications in Statistics: Theory and Methods 22, 1699-1707.

Arnold, B. C. 1996. Distributions with logistic marginals and/or conditionals, In: Distributions with Fixed Marginals and Related Topics (L. Rüschendorf, B. Schweizer, and M. D. Taylor (eds)), IMS Lecture Notes, Vol. 28, 15-32.

Arnold, B. C., Robertson, C. A. 1989. Autoregressive logistic processes. Journal of Applied Probability 26, 524-531.

Balakrishnan, N. (Ed.), 1992. Handbook of the Logistic Distribution. Marcel Dekker, New York.

Balakrishnan, N., Lai, C.-D. 2009. Continuous Bivariate Distributions, 2nd edition. Springer-Verlag, New York.

Banerjee. S., Gelfand, A. E. 2003. On smoothness properties of spatial processes. Journal of Multivariate Analysis 84, 85-100.

Bapat, R., Raghaven, T. E. S. 1997. Nonnegative Matrices and Applications. Cambridge University Press, Cambridge.

Chilés, J.-P., Delfiner, P. 1999. Geostatistics: Modeling Spatial Uncertainty. Wiley, New York.

Comellia, M., Féniès, P., Tchernev, N. 2008. A combined financial and physical flows evaluation for logistic process and tactical production planning: Application in a company supply chain. International Journal of Production Economics 112, 77-95.

Cramér, H. 1940. On the theory of stationary random processes. Annals of Mathematics 41, 215-230.

Cramér, H., Leadbetter, M. R. 1967. Stationary and Related Stochastic Processes: Sample Function Properties and Their Application. John Wiley \& Sons, New York.

Cressie, N. 1993. Statistics for Spatial Data. Wiley, New York.

Du, J., Ma, C. 2011. Spherically invariant vector random fields in space and time. IEEE Transactions on Signal Processing 59, 5921-5929. 
Dubey, S. 1969. A new derivation of the logistic distribution. Naval Research Logistics Quarterly 16, 37-40.

Ghosh, M., Choi, K. P., Li, J. 2010. A commentary on the logistic distribution, In: The Legacy of Alladi Ramakrishnan in the Mathematical Sciences, Part 3, 351-357. Springer-Verlag, New York.

Gikhman, I. I., Skorokhod, A. V. 1969. Introduction to the Theory of Random Processes. W. B. Saunders Co., Philadelphia.

Grasman, J. 1998. Stochastic epidemics: the expected duration of the endemic period in higher-dimensional models. Mathematical Biosciences 152, $13-27$.

Gerr, N. L., Allen, J. C. 1993. Stochastic versions of chaotic time series: Generalized logistic and Hénon time series models. Physica D: Nonlinear Phenomena 68, 232-249.

Hansen, E. R. 1975. A Table of Series and Products. Prentice-Hall, Englewood Cliffs, New Jersey.

Kotz, S., Balakrishnan, N., Johnson, N. L. 2000. Continuous Multivariate Distributions, Vol. 1: Models and Applications, 2nd edition. John Wiley \& Sons, New York.

Ma, C. 2005. Semiparametric spatio-temporal covariance models with the autoregressive temporal margin. Annals of the Institute of Statistical Mathematics 57, 221-233.

Ma, C. 2011a. Vector random fields with second-order moments or secondorder increments. Stochastic Analysis and Applications 29, 197-215.

Ma, C. 2011b. Covariance matrices for second-order vector random fields in space and time. IEEE Transactions on Signal Processing 59 (2011) 21602168.

Ma, C. 2011c. Covariance matrix functions of vector $\chi^{2}$ random fields in space and time. IEEE Transactions on Communications 59, 2554-2561.

Ma C. 2013. Mittag-Leffler vector random fields with Mittag-Leffler direct and cross covariance functions. Annals of the Institute of Statistical Mathematics 65, 941-958. 
Malik, H. J., Abraham, B. 1973. Multivariate logistic distributions. Annals of Statistics 1, 588-590.

Mansfield, E., Hensley, C. 1960. The logistic process: tables of the stochastic epidemic curve and applications. Journal of the Royal Statistical Society, Series B 22, 332-337.

Newman, T. J. Ferdy, J.-B., Quince, C. 2004. Extinction times and moment closure in the stochastic logistic process. Theoretical Population Biology $65,115-126$.

Pollard, H. 1946. The representation of $e^{-x^{\lambda}}$ as a Laplace integral. Bulletin of the American Mathematical Society 52, 908.

Silva, C. A., Sousa, J. M. C., Runkler, T., Palm, R. 2005. Soft computing optimization methods applied to logistic processes. International Journal of Approximate Reasoning 40, 280-301.

Sim, C. H. 1993. First-order autoregressive logistic processes. Journal of Applied Probability 30, 467-470.

Soboleva, T. K., Pleasants, A. B. 2003. Population growth as a nonlinear stochastic process. Mathematical and Computer Modelling 38, 1437-1442.

Tan, W. Y., Piantadosi, S. 1991. On stochastic growth processes with application to stochastic logistic growth. Statistica Sinica 1, 527-540.

Xue, Y., Xiao, Y. 2011. Fractal and smoothness properties of space-time Gaussian models. Front. Math. China 6, 1217-1248.

Yaglom, A. M. 1987. Correlation Theory of Stationary and Related Random Functions, Vol. I: Basic Results. Springer-Verlag, New York. 